\title{
Le sport dans la civilisation étrusque : entre Grèce et Rome
}

Sport in the Etruscan Civilization: between Greece and Rome

Jean-Paul Thuillier

\section{(2) OpenEdition}

Journals

Édition électronique

URL : http://journals.openedition.org/etudesbalkaniques/165

ISSN : 2102-5525

Éditeur

Association Pierre Belon

Édition imprimée

Date de publication : 1 janvier 2004

Pagination : 13-32

ISBN : 2-910860-11-6

ISSN : $1260-2116$

\section{Référence électronique}

Jean-Paul Thuillier, «Le sport dans la civilisation étrusque : entre Grèce et Rome », Études balkaniques [En ligne], 11 | 2004, mis en ligne le 06 avril 2009, consulté le 30 avril 2019. URL : http:// journals.openedition.org/etudesbalkaniques/165

Ce document a été généré automatiquement le 30 avril 2019.

Tous droits réservés 


\title{
Le sport dans la civilisation étrusque: entre Grèce et Rome
}

\author{
Sport in the Etruscan Civilization: between Greece and Rome
}

Jean-Paul Thuillier

1 Un des mots qui revient le plus souvent, et depuis très longtemps, à propos des Etrusques est celui de «mystère » : s'agissant de cette civilisation qui fut la première d'Italie et qui trouva son apogée aux VII ${ }^{\mathrm{e}}$ et $\mathrm{VI}^{\mathrm{e}}$ s. av. J.-C., ce mot n'est pas sans danger car il peut ouvrir la porte aux explications les plus fantaisistes ou même les plus fantasmagoriques, mais il résume assez bien, quoique de façon un peu abrupte, les nombreuses ignorances qui sont encore les nôtres aujourd'hui. Il suffit de rappeler les problèmes délicats que pose toujours l'interprétation des longs - et rares - textes étrusques qui nous sont parvenus, et, à côté de celle de la langue, la question des origines de ce peuple reste ouverte, même si nous préférons aujourd'hui mettre l'accent sur la formation et l'histoire des Etrusques tout au long du premier millénaire av. J.- $\mathrm{C}^{1}$.

En revanche, dans le tableau général de nos connaissances sur cette civilisation, un thème comme celui des jeux et des sports étrusques a toujours été considéré comme assez privilégié. Voici par exemple ce qu'écrivait il y a une quarantaine d'années Jacques Heurgon dans son très beau livre sur la vie quotidienne chez les Etrusques : «Ce que nous savons peut-être de plus clair sur les mœurs des Etrusques, c'est à leurs jeux que nous le devons, parce que, sous la forme particulière de jeux funèbres, ils ont aimé les peindre sur les murs de leurs tombes ou les sculpter sur leurs cippes et leurs sarcophages $»^{2}$. Rappelons que ces peintures de l'Etrurie antique sont d'autant plus intéressantes que la grande peinture grecque correspondante sur le plan chronologique a entièrement disparu : c'est parce que les anciens Toscans ont eu l'heureuse idée de décorer ainsi leurs hypogées, leurs tombes souterraines creusées dans le roc, que ces peintures ont été conservées, tout au moins jusqu'à l'ouverture des tombes et que les apports extérieurs ne commencent alors leur œuvre de destruction. Stendhal parlait au XIX ${ }^{\mathrm{e}}$ s. des petites caves peintes du Père-Lachaise de Corneto (nom médiéval de l'actuelle Tarquinia, qui a ainsi 
retrouvé son nom antique) $)^{3}$. Et c'est très justement que ces fresques tarquiniennes constituent le pôle d'attraction touristique principal : après tout, comme le rappelait le grand étruscologue Massimo Pallottino, nous ouvrons là le premier chapitre de l'histoire de la peinture italienne.

Et pour en venir donc à la question iconographique, si l'on prend l'exemple des fresques funéraires des $\mathrm{VI}^{\mathrm{e}}$ et $\mathrm{V}^{\mathrm{e}}$ s., bien représentées à Tarquinia mais aussi à Chiusi, on constate en effet avec J. Heurgon que les motifs sportifs sont en très bonne place, à côté des scènes de banquet et de danse. Quelle est d'ailleurs la signification de ces images ? C'est là un problème plus complexe qu'il n'y paraît au premier abord et qui a suscité de nombreux débats. Il est difficile de ne pas penser d'emblée que les peintures de ces hypogées évoquent des jeux organisés lors des funérailles du titulaire de la tombe : les Etrusques ont manifestement connu un rituel comme celui que nous décrit déjà Homère lors des funérailles de Patrocle au chant XXIII de l'Iliade, avec diverses compétitions hippiques la course de biges est le moment le plus important - et athlétiques - parmi ces dernières, le pugilat et la lutte. Nous nous trouvons là devant une phase d'un rite de passage qui concerne le mort certes mais aussi les vivants : le clan familial et même au-delà les voisins et les habitants de la cité sont éprouvés et même un moment défaits par ce deuil qu'il s'agit de surmonter. Les compétitions, les danses, le banquet permettent au groupe de passer cette épreuve psychologique, de se restaurer dans tous les sens du terme et de retrouver des forces pour assurer l'avenir de leur communautét.

Quant au défunt lui-même, il peut trouver un réconfort dans les peintures qui prolongent d'une certaine façon l'efficacité rituelle des jeux, et il peut se réjouir de cette vision, si l'on estime qu'il vit d'une vie ralentie dans l'au-delà. Quand on voit, dans deux tombes de Tarquinia (la Tombe des Augures et la Tombe justement dite des Inscriptions) datant des années 530, que le nom de certains athlètes, lutteurs ou boxeurs, est peint à côté de leur image, on peut se demander s'il ne s'agissait pas des vedettes favorites du défunt titulaire de l'hypogée : et l'on songe alors à Trimalcion, le célèbre parvenu du Satiricon de Pétrone, qui, six siècles après, demande à l'entrepreneur chargé de construire son tombeau, de peindre sur celui-ci les combats de Pétraitès, son gladiateur favori, "afin d'avoir le bonheur de vivre après sa mort $»^{5}$. Nul doute que certains supporters de football aimeraient emporter dans leur tombe l'image de Zidane ou de Beckham si cela se faisait encore aujourd'hui. Des sociologues ont cité le cas de cette femme « tifosa » de la Juventus de Turin qui ne désirait qu'une seule chose dans son éloge funèbre: qu'on dise d'elle qu'elle avait été une fidèle supportrice de la «Juve »!

Mais la présence de vedettes sportives sur ces fresques funéraires de Tarquinia avait sans doute aussi pour but de mettre l'accent sur le faste de ces cérémonies, sur les sommes qu'elles avaient coûtées, et donc sur le statut social des familles concernées. Il s'agissait sans aucun doute aussi de souligner le rang social du défunt et de sa gens : ce n'est pas par hasard que, dans la grande nécropole des Monterozzi, aujourd'hui située à la sortie du bourg moderne de Tarquinia, seules $2 \%$ des tombes sont peintes. Il s'agissait là d'un investissement qui n'était pas à la portée de toutes les bourses et c'était encore plus vrai des jeux eux-mêmes qui constituent donc un des motifs fréquents sur les parois peintes. On peut même parfois aller au-delà de cette constatation. Dans une autre tombe de Tarquinia, la Tombe des Biges, qui est un peu plus récente que les deux précédentes déjà citées, on voit en effet un motif rare : des tribunes de bois qui sont remplies d'un public nombreux et varié, avec des hommes et des femmes, et même, allongés sous les tribunes, de jeunes esclaves qui sont en train de se livrer à des jeux érotiques. Le nombre de ces 
spectateurs, qui assistent donc à des épreuves hippiques et athlétiques, semble révéler qu'on n'est pas en présence d'une seule famille : on est là au-delà de la seule sphère du privé et il n'est pas interdit d'imaginer que le noble défunt était un magistrat de sa cité de Tarquinia, et que ses descendants ont organisé des jeux funéraires auxquels étaient conviés une partie au moins de ses «administrés». En tout cas, c'est bien la position sociale du mort et de sa famille qui est ici une fois de plus exaltée ${ }^{6}$.

Certains historiens s'appuient sur le témoignage d'Hérodote pour ne faire remonter les origines des jeux étrusques qu'au milieu du $\mathrm{VI}^{\mathrm{e}}$ s. avant J.-C. environ. L'historien grec nous a en effet transmis l'anecdote suivante à propos des habitants de la ville de CaeréAgylla : ces derniers, alliés aux Carthaginois, avaient remporté dans les années 540 une bataille navale, au large d'Alalia, sur les Phocéens et avaient ensuite lapidé une partie de leurs prisonniers. Le lieu de ce massacre devint maudit et toute une série de prodiges s'ensuivit : il fallait procéder à une purification et les Agylléens allèrent consulter l'oracle de Delphes.

7 «La Pythie leur ordonne de faire ce que de nos jours ils accomplissent encore : ils offrent aux mânes des Phocéens de riches sacrifices, et ils instituent en leur honneur des jeux gymniques et équestres ${ }^{7}$. Comme on le voit, les éléments helléniques ne manquent pas dans ce tableau et l'Etrurie apparaît d'emblée en ce domaine comme la débitrice de Delphes et même comme une simple province de l'Hellade. Et sur le plan iconographique, pour ne citer que ce seul exemple, la présence de nombreux motifs de jeux athlétiques sur les fresques funéraires de Tarquinia appartenant à la seconde moitié $\mathrm{du} \mathrm{VI}{ }^{\mathrm{e}}$ s., paraît apporter une confirmation immédiate à cette indication de l'historien grec.

« Il faut pourtant remonter plus loin en arrière. Tite-Live, dans son récit du règne de Tarquin l'Ancien, note que celui-ci, pour fêter sa victoire sur les Latins, organisa - dans un Grand Cirque nouvellement aménagé - des jeux plus somptueux que ses prédécesseurs (peut-être Tite-Live songe-t-il, en disant cela, aux Consualia qu'aurait fondés Romulus ?). Le spectacle (ludicrum) comprenait à l'affiche des chevaux (equi) et des boxeurs (pugiles) " qu'on avait fait venir principalement d'Etrurie » (ex Etruria maxime acciti ${ }^{8}$ ) Ainsi, dans certaines cités étrusques tout au moins, des épreuves athlétiques étaient - elles connues dès la fin du VII ${ }^{e}$ siècle avant notre ère : on pensera par exemple à la cité la plus proche de Rome, Véies, l'éternelle rivale, qui devait s'opposer à l'Urbs non seulement pour la conquête de terres mais aussi et peut-être surtout pour la main-mise sur les salines situées aux bouches du Tibre. La maîtrise du sel, ce "pétrole de l'Antiquité ", était en effet essentielle pour ces populations d'agriculteurs. Toujours est-il qu'un vase de bucchero incisé, trouvé à Véies au siècle dernier, et datant du dernier tiers du VII siècle avant notre ère, vient tout de suite illustrer cette proposition : à côté des différents motifs d'animaux réels ou fantastiques, on peut en effet observer sur la panse de cette olla une très belle scène de pugilat, avec deux boxeurs affrontés, la garde haute, et apparemment vêtus d'un maillot-tunique. Mais Véies n'est pas l'unique cité étrusque impliquée : à Caeré, l'actuelle Cerveteri, déjà citée plus haut, on a découvert une urne peinte, également du VII ${ }^{\mathrm{e}}$ s., qui représente un match de boxe ${ }^{9}$, et on connaît même aujourd'hui, pour la même période et la même cité (tombe de San Paolo), une olpè de bucchero - cette céramique noire typique de l'Etrurie - qui est décorée de scènes mythologiques avec Médée et Dédale, et Jason peut-être, engagé en tout cas dans un pugilat ${ }^{10}$. Enfin, et toujours pour le $\mathrm{VII}^{\mathrm{e}}$ s., une olla peinte attribuée au Peintre de Civitavecchia, qui 
travaillait peut-être dans la même région de Caeré, nous permet d'assister à un combat de boxe qui est pour la première fois accompagné par un musicien, un aulète : ce sera désormais, nous le verrons plus loin, une constante dans cette civilisation ${ }^{11}$.

9 La boxe n'est certes pas la seule compétition figurée dès cette haute époque en Etrurie, puisqu'un petit groupe de bronze récemment trouvé à Murlo, au sud de Sienne, et datant de la fin du VII ${ }^{\mathrm{e}}$ s. également, représente deux lutteurs en pleine action sous le regard d'un arbitre armé d'une longue baguette, mais il n'en reste pas moins que le pugilat sera toujours le sport favori des Etrusques - avec les courses de chars bien entendu - ...ainsi que celui des Romains. (Et la réunion de ces deux spécialités, équestre et pugilistique, n'est pas sans faire penser d'ailleurs au patronage des Dioscures, maîtres et protecteurs de ces deux disciplines sportives respectives). La prédominance du pugilat se vérifie par le nombre des représentations aux $\mathrm{VI}^{\mathrm{e}}$ et $\mathrm{V}^{\mathrm{e}} \mathrm{s}$, , en particulier sur les fresques de Tarquinia, où les boxeurs sont parfois les seuls athlètes figurés, et où ils occupent souvent une place de choix, encadrant par exemple la porte d'entrée comme deux gardiens venant menacer l'éventuel visiteur indésirable (Tombe Cardarelli) ${ }^{12}$.

10 Cette popularité de la boxe peut être aussi constatée à partir de l'examen des stèles funéraires de Felsina (Bologne) : elle est d'abord le seul sport athlétique représenté parfois à côté de la course de biges, et l'on retrouve alors une fois de plus le programme sportif défini par Tite-Live à propos de Tarquin l'Ancien. Mais ce sport occupe également une place privilégiée dans la décoration : on peut relever en particulier le cas de la stèle $n$ - 169 (d'après le catalogue de $\mathrm{P}$. Ducati), où la scène de pugilat s'étale entièrement sur une zone figurée, avec les cinq mêmes personnages, disposés selon un schéma différent, que l'on va identifier sur la panse de l'amphore B 64 du Peintre de Micali (les deux athlètes, le soigneur à l'éponge, le musicien et l'arbitre) ${ }^{13}$.

11 Lorsque l'artiste a en effet voulu figurer un festival sportif de façon aussi complète que possible, montrant aussi bien la procession que les ludi hippiques et gymniques, comme sur l'amphore à figures noires du Peintre de Micali, conservée au British Museum (B 64), il n'a pas manqué de donner un relief tout particulier à la scène de pugilat, laquelle écrase, par sa situation et le nombre des personnages présents, le reste des compétitions ${ }^{14}$. Il est intéressant de constater à ce sujet que le tibicen, l'aulète (le flûtiste, comme nous disons souvent à tort en français, puisqu'il s'agit d'un instrument à anche), que ce musicien donc est presque toujours présent à côté des boxeurs étrusques ${ }^{15}$. Plusieurs auteurs anciens ont d'ailleurs relevé cette coutume, qui choquait certains esprits chagrins : on voulait en effet voir là un des signes de cette mollesse, de cette vie dissolue que l'on reprochait souvent aux Etrusques dans l'Antiquité, en particulier chez les auteurs grecs. En réalité, cette accusation de «truphê » - pour reprendre le terme hellénique - ne faisait que traduire la jalousie de ces mêmes Grecs à l'égard d'un peuple étrusque qui jouissait de conditions économiques très favorables et qui, ô scandale, permettait même aux femmes de jouer un rôle non-négligeable dans la vie sociale. Reprendre ce grief de "truphê » à propos de l'accompagnement musical du pugilat étrusque était particulièrement injuste et stupide : comme on le voit bien encore aujourd'hui par la boxe traditionnelle thaillandaise, qui n'a rien de particulièrement doux, il s'agissait pour les musiciens de rythmer les assauts des deux adversaires et même de réveiller leur ardeur au combat, si celle-ci faiblissait par trop.

12 A côté des données chronologiques ou chiffrées, d'autres constatations révèlent encore l'importance du pugilat dans les mœurs sportives des Etrusques : on a pu identifier, sur 
un relief archaïque de Chiusi, l'existence d'un véritable ballet de la boxe, avec trois athlètes boxant et dansant en cadence, sous la direction, encore plus nécessaire ici, d'un aulète (et peut-être est-ce cette alliance entre boxe et danse qui est à l'origine de ces accusations grecques de " truphê » évoquées à l'instant... ${ }^{16}$. On ne saurait trouver en tout cas d'illustration plus précise à l'expression de Jean Cocteau qui qualifiait les boxeurs de «danseurs qui tuent» et on notera là encore que de telles chorégraphies ne sont pas ignorées aujourd'hui - on pensera par exemple au ballet K.O.K. de Régine Chopinot. Avant de découvrir une scène de boxe classique sur un relief de Chiusi inédit jusqu'à une époque récente ${ }^{17}$, on aurait pu trouver surprenant de rencontrer un tel document à Chiusi, où l'on manifestait surtout un grand engouement pour la lutte, et où l'on assiste souvent, sur les reliefs comme sur les fresques, à une prise très spectaculaire qui voit un des adversaires accomplir un véritable vol plané au-dessus de l'autre concurrent: ce mouvement sportif, qui mettait fin à une des phases du match - si les Etrusques ont repris la règle grecque des trois « tombers » - apparait en tout cas comme une signature, comme un « motivo-firma » des artisans de Chiusi. Il faut en effet tenir compte ici de la spécificité des cités étrusques qui, pour avoir tissé de nombreux liens au sein de la dodécapole, ont su cependant garder leur originalité dans bien des domaines.

13 Nous n'avons cité jusqu'ici que deux compétitions, la boxe et la lutte, qui ne sont plus considérées aujourd'hui comme des disciplines relevant de l'athlétisme : mais il n'en était pas ainsi dans l'Antiquité, où ces deux épreuves dites "lourdes" ou "pesantes" appartenaient bien aux jeux gymniques ou athlétiques au même titre que les épreuves légères du pentathlon. Les Etrusques ont certes connu ces dernières - mais un peu plus tard, si l'on se réfère à la seule iconographie ; un bon exemple nous est donné par la tombe peinte des Olympiades, datant des années 530 environ, et qui a été découverte en 1958 à Tarquinia, grâce aux méthodes modernes de détection de la Fondation Carlo Lerici de Milan ${ }^{18}$.

14 Sur les deux parois principales de cette petite tombe, on peut voir d'une part une course de biges et un combat de boxe - cette dernière scène est très abîmée, mais on retrouve là un autre exemple de ce programme que nous connaissons bien désormais - et d'autre part un ensemble de trois épreuves, avec des coureurs à pied, un sauteur en longueur et un discobole - et cette seconde paroi offre aussi le jeu typiquement étrusque du Phersu : un homme encapuchonné et armé d'une massue est attaqué par un chien féroce qu'excite un bourreau masqué. Ce dernier porte le nom de Phersu, c'est-à-dire le «Masque » (un mot qui correspond au latin persona). Certains ont voulu voir là, à tort, la préfiguration des combats romains de gladiateurs qui semblent en réalité trouver leur origine non pas en Etrurie mais en Campanie, comme le montrent bien des peintures funéraires de Paestum ${ }^{19}$.

15 La réunion des trois épreuves athlétiques citées plus haut pourrait laisser supposer que les Etrusques connaissaient aussi le pentathlon à la grecque, lequel comprenait encore lutte et lancer du javelot.: de même, sur certaines amphores panathénaïques, c'est un simple choix d'épreuves qui est représenté pour une victoire au pentathlon. Et cette impression est confirmée par d'autres documents comme l'amphore B 64 du Peintre de Micali déjà citée, où l'on peut voir côte à côte un discobole et un lanceur de javelot, et surtout un relief archaïque de Chiusi, conservé à Palerme, sur lequel un même athlète, au moment de la remise des récompenses, tient à la fois un disque et un javelot. Mais, sur un tel sujet, nous devons rester dans l'incertitude, en raison de l'absence de textes littéraires ou d'inscriptions - à supposer que nous puissions comprendre de tels documents 
épigraphiques en étrusque, et que nous sachions même que tel texte évoque des règles sportives! Seul l'excellent Z. Mayani arrivait à déchiffrer sans problème les « règles de la lutte étrusque, dûment approuvées par le syndicat national des lutteurs $»^{20} \ldots$

On n'aura pas l'audace non plus de décrire en détail le déroulement de certaines épreuves dont nous supposons simplement qu'elles étaient plus ou moins identiques à celles des Grecs. Les coureurs à pied de la Tombe des Olympiades sont en train de sprinter : mais disputent-ils un stade? Sont-ils à la fin d'une course de fond? Quelle était alors la distance de ce dolichos, et y avait-il aussi en Etrurie une épreuve de diaulos ${ }^{21}$ ? Les images nous permettent certes de constater que le sauteur en longueur étrusque utilisait habituellement des haltères pour améliorer sa performance, que le lanceur de javelot propulsait son instrument grâce à une courroie qu'on appelle en latin l'amentum (et l'on en voit une très belle représentation sur les fresques de la Tombe du Singe à Chiusi). Tout cela est également présent chez les Grecs et l'on peut d'ailleurs penser, à voir la maladresse avec laquelle les artistes étrusques montrent par exemple le geste du discobole, que ce dernier exercice n'était pas très en faveur en Etrurie : ne faut-il pas penser la même chose des autres épreuves du pentathlon (lutte exceptée évidemment) ?

On serait cependant tenté de noter un changement à partir du milieu du $\mathrm{V}^{\mathrm{e}}$ s. environ. Nombreux sont en particulier les bronzes, ex-voto ou décorations de candélabres par exemple, qui représentent à cette époque un athlète de type pentathlonien. On connaît même un athlète qui ne peut guère être qu'un lanceur de poids: cette compétition existait-elle en Etrurie? Mais il n'est pas aisé de savoir si cela correspond à la réalité sportive de l'Etrurie ou si ce sont seulement des motifs esthétiques ou fonctionnels qui entrent ici en jeu : il est plus facile de placer un discobole au sommet d'un candélabre qu'un match de boxe intégral. Dans certains cas, des particularismes locaux peuvent aussi être envisagés : on a constaté par exemple le nombre tout à fait remarquable de sujets athlétiques pentathloniens sur les bronzes de Spina, ce qui conduit J.-R. Jannot à s'interroger ainsi : « Les lanceurs de disque ou de javelot, les athlètes utilisant le strigile, les sauteurs, témoignent de la faveur des épreuves grecques dans des proportions qui n'ont pas de commune mesure avec celles des autres contrées de l'Etrurie. On mesure l'importance de cette thématique dans le port des bouches du Pô. Serions-nous en face d'une culture périphérique plus réceptive aux influences helléniques ${ }^{22}$ ?

Tout en ne négligeant pas ces influences, il est important d'insister sur le fait que les jeux athlétiques étrusques ne sont pas un simple décalque des agônes helléniques: sur ce point comme sur d'autres, l'originalité étrusque n'est pas à sous-estimer. Nous avons déjà vu l'environnement musical spécifique de la boxe toscane, mais au-delà des détails techniques, d'autres différences plus sensibles doivent être soulignées. La question de la nudité pourrait être au premier abord un critère essentiel, puisque les Grecs eux-mêmes revendiquaient cette nudité athlétique comme un trait qui les distinguait radicalement des Barbares. En fait, sur ce point, il n'en est rien en ce qui concerne la société étrusque puisqu'on peut rencontrer par exemple des lutteurs étrusques entièrement nus, comme dans la Tombe des Augures de Tarquinia, qui date de 540-530 av. J.-C. (et, à analyser de près textes littéraires et documents iconographiques, on pourrait même se demander mais la question est sacrilège... - si les Etrusques n'ont pas précédé leurs « maîtres » grecs en cette occasion ${ }^{23}$ !).

19 Le réalisme de l'art étrusque, en tout cas à la fin du $\mathrm{VI}^{\mathrm{e}}$ et au début du $\mathrm{V}^{\mathrm{e}}$ s., permet de surcroît de mieux comprendre certaines pratiques que l'idéalisation de l'athlète, dans 
l'art attique contemporain, a presque totalement occultées. Ainsi constate-t-on par exemple que certains athlètes étrusques sont munis d'une ceinture "athlétique » et même plus précisément d'un "suspensoir »: le sexe est tenu relevé par une cordelette elle-même rattachée à une ceinture. Les fresques de la Tombe du Singe à Chiusi offrent des exemples particulièrement nets de cette pratique; et dans certains cas les témoignages sont perdus: ainsi, pour rester à Chiusi, certains détails de ce type qui étaient peints sur les reliefs archaïques sont aujourd'hui effacés. A vrai dire, il n'en allait pas autrement dans la réalité pour les Grecs : d'ailleurs, quelques - très rares - peintures de vases, dont cependant un beau cratère d'Euphronios, viennent le confirmer ${ }^{24}$. Les athlètes grecs avaient les mêmes impératifs physiologiques et devaient donc pour la très grande majorité porter un appareil du type suspensoir. Ce qui n'empêchait pas que, du point de vue de la décence, ils fussent nus. Reste donc que sur cette question, les Etrusques n'étaient pas de vrais barbares ; mais nous avons aussi par là la preuve que les artistes étrusques ne se contentaient pas de reproduire des images grecques, comme on le suppose trop souvent, mais qu'ils montraient bel et bien les réalités locales.

\section{Epreuves hippiques} celui des courses hippiques, et surtout celui des courses de chars qui ont connu là, comme plus tard à Rome, un succès fantastique. Nous avons déjà vu que vers 610-600 av. J.-C., le roi Tarquin l'Ancien avait fait venir d'Etrurie des chevaux et des pugilistes. On ne s'attardera pas longtemps sur les représentations étrusques les plus anciennes: mais un peu après 600 , des plaques de terre cuite, mises au jour dans une résidence seigneuriale à Murlo, nous montrent une course de chevaux montés à cru par des jockeys qui se dirigent vers le prix de la compétition, un chaudron juché sur une colonne. Murlo est à quelques kilomètres au sud de Sienne : c'est donc une sorte de Palio avant la lettre auquel nous assistons ${ }^{25 !}$

21

Mais là encore, ce sont les fresques funéraires de Tarquinia ou de Chiusi qui nous offrent les attestations les plus frappantes. Revenons à la Tombe des Olympiades dont le nom contribue malencontreusement à cette impression d'hellénisation généralisée en Etrurie. Sur la paroi gauche de ce petit hypogée, à côté des boxeurs, quatre biges se précipitent vers la borne d'arrivée, un simple poteau rouge : le cocher de tête se retourne pour voir où en sont ses adversaires, et le quatrième est victime d'un "naufrage ", autrement dit d'une chute spectaculaire. La tenue de ces cochers est tout à fait significative : ils portent tous une tunique courte qui arrive à mi-cuisse et pour certains d'entre eux ont un casque de cuir. Cette tenue n'a rien à voir avec celle des cochers grecs, qui, tels l'Aurige de Delphes, ont une longue tunique qui tombe à leurs pieds et ont normalement la tête nue. Par ailleurs, la technique de conduite appelle les mêmes remarques: les cochers étrusques ont, pour ne pas les perdre, les guides nouées autour de la taille - un nœud qui apparaît ici quelque peu démesuré - cependant que les cochers grecs tiennent simplement les guides dans les mains - un « peloton » de guides tombant dans la caisse du char permet là aussi de mieux les contrôler. Si l'on ajoute que les cochers étrusques manient un fouet, cependant que leurs collègues grecs ont un long aiguillon, le kentron, on constate qu'il y a un fossé entre les cultures hippiques de ces deux peuples ${ }^{26}$. En revanche, qu'il s'agisse de l'équipement ou de la technique de ménage du char, les Romains ont pratiquement tout appris des Etrusques... et très peu des Grecs : des milliers

Études balkaniques, 11 | 2008 
de documents, grands et petits, mosaïques, bas-reliefs, terres cuites, intailles, confirment cette constatation.

Une impression qui va encore se renforcer si l'on se penche maintenant sur le type des chars utilisés dans ces compétitions équestres. Nous avons parlé, à propos de ces fresques, de biges, c'est-à-dire de chars attelés à deux chevaux : il est curieux de voir que les Etrusques ne connaîtront jamais en course le quadrige qui est le char-roi en Grèce, où la victoire en quadrige est en fait réservée à une élite sociale et politique : on sait par exemple que l'Aurige de Delphes courait pour le compte d'un tyran sicilien. En revanche, les Etrusques ont une prédilection pour le trige, le char attelé à trois chevaux, avec deux chevaux timoniers et un cheval extérieur libre : ainsi, sur les reliefs archaïques de Chiusi, presque la moitié des courses attelées sont des courses de triges ${ }^{27}$. Et là c'est presque un cas d'école : en effet, les Grecs n'ont jamais organisé de courses de triges, alors que les Romains vont reprendre cette épreuve à leur compte, comme on le voit bien chez Denys d'Halicarnasse et par plusieurs inscriptions livrant les palmarès de cochers vedettes. C'est si vrai qu'il y avait à Rome, au Champ-de-Mars, le long du Tibre, un cirque d'entraînement, qu'on appelait le Trigarium, en raison bien sûr des triges qui s'y produisaient : il est certain que ce lieu ainsi baptisé remontait à la période " étrusque » de Rome, sous les Tarquins ${ }^{28}$. On versera un dernier argument dans ce débat : c'est l'intérêt des Etrusques et des Romains pour les épreuves de cavaliers-acrobates (en latin, les desultores), qui sautaient à bas de leur monture à tel ou tel moment de la course, ou qui sautaient d'un cheval sur l'autre, alors que les uns et les autres n'ont presque jamais montré de courses simples avec des jockeys classiques (après le cas de Murlo, on ne trouve plus de telle représentation en Etrurie ${ }^{29}$. Il y a là un nouveau point de contact entre sports étrusque et romain, et une nouvelle divergence par rapport à la réalité hellénique.

\section{Edifices de spectacle : du stade au cirque}

Si l'on examine maintenant le cadre dans lequel se déroulaient de telles compétitions sportives, on va encore distinguer nettement les deux civilisations, grecque et étrusque, du point de vue sportif. On sait qu'en Grèce - et il suffit encore une fois de penser aux grands sites panhelléniques comme Olympie ou Delphes - les jeux gymniques avaient lieu dans le stade, cependant que les jeux hippiques étaient disputés dans l'hippodrome : ces deux « lieux » - on hésitera à parler d'édifices, en particulier pour l'époque archaïque pouvaient d'ailleurs être séparés par une grande distance, comme on le voit à Delphes où l'hippodrome restera dans la plaine d'Itéa quand le stade sera transféré dans la partie la plus haute du sanctuaire, au pied de la roche Phédriade Rhodini ${ }^{30}$. A Olympie, les deux lieux étaient très proches, mais l'hippodrome a presque entièrement disparu, emporté par les divagations médiévales du fleuve Alphée.

La situation en Etrurie, telle qu'elle apparait sur les peintures ou les reliefs, est au contraire de type romain: compétitions équestres et athlétiques sont manifestement regroupées sur un même espace. Pensons simplement à l'exemple de la Tombe des Olympiades, déjà cité, où boxeurs et course de biges sont situés sur la même paroi, sans la moindre séparation. Et ce sera le cas dans le circus romain lors des ludi traditionnels, qui suivent l'exemple des origines : Tarquin l'Ancien « crée » le Circus Maximus, entre Palatin et Aventin, dans la vallée Murcia aménagée, et inaugure l'édifice par un ludicrum 
composé de pugiles et d'equi. Il faudra attendre la fin du $1^{\mathrm{er}}$ s. apr. J.-C. et l'hellénisation croissante des spectacles romains, pour assister, sur le site de l'actuelle Place Navone, et c'est un des plus beaux exemples de permanence urbanistique, à la construction du stade de Domitien destiné à accueillir les certamina graeca que Néron avait déjà essayé d'introduire dans l'Urbs.

On n'a pas retrouvé jusqu'à présent de "circus » étrusque et cela n'a certes rien d'étonnant, étant donné le caractère léger et provisoire des installations qui devaient être mises en place pour des jeux, en particulier au $\mathrm{VI}^{\mathrm{e}} \mathrm{s}$. Mais il n'est pas interdit de faire des hypothèses sur l'emplacement de ces cirques : ceux-ci ont toutes chances par exemple de se trouver dans la plaine la plus proche du plateau de tuf, sur lequel sont bâties la plupart des grandes cités de l'Etrurie méridionale, Caeré, Tarquinia, Orviéto...; et si cette plaine est de surcroît voisine d'une des grandes nécropoles de la cité, là où se déroulaient les funérailles et les jeux funèbres, l'hypothèse acquiert encore de la crédibilité. Plus tard, le cirque romain sera souvent situé aussi à proximité d'une grande nécropole, assez loin du centre de la ville pour des raisons d'espace évidentes. On peut faire d'autres observations à partir des fouilles archéologiques. Et constater tout d'abord que dès le VII ${ }^{\mathrm{e}}$ s., plusieurs tombes orientalisantes de Tarquinia présentent une structure qu'on a pu qualifier de «théâtriforme »: un dromos très large, semblable à une petite place, est souvent bordé, sur plusieurs côtés, de gradins destinés à accueillir des spectateurs qui pouvaient ainsi regarder des danses scéniques mais aussi des matches de boxe ou de lutte, dans le cadre bien entendu de jeux funéraires. Plus tard, au $\mathrm{VI}^{\mathrm{e}}$ s., d'autres tombes, à Bléra ou Vulci, présentent encore des structures du même type ${ }^{31}$.

Mais ce sont les fresques qui nous offrent encore la plus belle représentation d'installations pour des spectateurs avec la Tombe des Biges de Tarquinia, datée des années 500 et que nous avons déjà citée. On peut voir là de remarquables tribunes en bois protégées par des vela, et qui semblent de surcroît illustrer directement la description du Grand Cirque au temps du roi étrusque Tarquin l'Ancien, telle qu'elle nous est faite par Tite-Live ou encore Denys d'Halicarnasse ${ }^{32}$. Pendant que de jeunes serviteurs se livrent, étendus sous les tribunes, à des jeux plus ou moins innocents, de «nobles» spectateurs sont assis sur des bancs, hommes et femmes mêlés; la mixité de ce public est un trait éminemment significatif, d'autant qu'en un cas au moins, c'est une femme qui semble occuper le premier plan, sinon la place d'honneur dans une tribune (ce qui n'est pas une raison suffisante pour parler, comme on l'a fait trop longtemps, de matriarcat étrusque). Tout cela nous éloigne bien sûr des stades grecs, et par exemple de celui d'olympie où aucune spectatrice n'était accueillie, à l'exception de la prêtresse de Démèter Chamynè ; et cela nous rapproche encore une fois de Rome dont le Grand Cirque était, selon Ovide, un lieu privilégié pour les tentatives de séduction ${ }^{33}$ !

L'accent qui est mis sur le caractère spectaculaire des jeux étrusques ne se retrouve certainement pas en Grèce, où l'on note, non sans une certaine surprise, que le stade d'olympie ne sera jamais équipé de gradins pour les spectateurs: c'est là un trait symbolique pour ce haut-lieu de l'agonistique grecque, et cela nous amène directement à une autre réflexion concernant cette fois la condition sociale des athlètes étrusques. Comme le montrent bien les quelques sources littéraires et épigraphiques que nous pouvons utiliser, ainsi que les documents iconographiques, qui font se côtoyer sans vergogne sportifs, bourreaux et bouffons, ce n'est pas le modèle agonistique grec de l'époque classique, qui était appliqué en Etrurie. En effet, ce ne sont pas des " éphèbes » 
issus pour la plupart des meilleures familles que nous voyons évoluer sur le sable de la piste, ou l'herbe de la plaine; ce sont des professionnels, des « dépendants » rattachés en général à la troupe d'un seigneur, d'un lucumon peut-être - si ce mot bien connu n'est pas seulement un nom propre - comme celle de ce roi de Véies qui, à la fin $d u V^{\mathrm{e}}$ s., l'avait retirée des jeux panétrusques de Volsinies, furieux qu'il était de ne pas avoir été élu sacerdos de la ligue par ses pairs. Cette anecdote, que nous a transmise Tite-Live ${ }^{34}$, semble surtout concerner les artistes de la scène (artifices), mais il est clair que les athlètes, que l'on ne distingue d'ailleurs pas toujours très bien des premiers, n'étaient pas dans une condition différente. Il paraît difficile par ailleurs de discerner des évolutions chronologiques et des disparités géographiques profondes quant à ce point. Enfin, cette condition sociale inférieure des sportifs étrusques rend plus que problématique l'hypothèse, imprudemment avancée sur la foi d'une inscription mutilée, d'une participation étrusque à de grands concours panhelléniques comme ceux de Delphes ${ }^{35}$.

Les Etrusques étaient, selon un mot bien connu de Tite-Live, les "plus religieux des hommes » et ils ne risquaient donc pas d'échapper à une situation qui est celle de toutes les sociétés méditerranéennes antiques: aussi nous faut-il encore revenir sur une dimension essentielle des jeux étrusques, qui est la dimension religieuse. Nous avons déjà évoqué ces jeux funèbres dont les images constituent l'essentiel de notre documentation, et qui soulignent le statut élevé de certains défunts. On retrouve aussi là des illustrations de la condition particulière de la femme étrusque. Sur des fresques (Tombe du Singe, Chiusi) ou sur une stèle de pierre (Bologne-Felsina), le défunt était plutôt une défunte : ainsi non seulement les femmes assistaient-elles aux compétitions sportives, mais cellesci pouvaient aussi être célébrées lors de leurs funérailles.

Mais dans l'Antiquité classique, selon une distinction habituelle, il y avait, à côté des ludi funèbres, des ludi sacri, qui étaient offerts à diverses divinités, et les dieux se réjouissaient allègrement de ces spectacles : l'organisation de jeux pour les apaiser, par exemple en cas d'épidémie, la conception des ludi comme rituel de procuration semble bien être une tradition italique, étrusque d'abord puis largement diffusée à Rome - et contrairement à ce que laisse entendre le texte d'Hérodote que nous avons cité au début, l'oracle de Delphes n'avait pas du tout la même politique religieuse sur ce point. Cette source littéraire nous fait d'ailleurs connaître au passage un exemple de jeux parfaitement publics : c'est bien la cité de Caeré qui organise ces jeux après la bataille d'Alalia et la lapidation des prisonniers phocéens. On peut aussi supposer sans grand risque d'erreur que la plupart des cités étrusques célébraient - annuellement ? - des jeux, en l'honneur au moins de leur divinité poliade, sur le modèle des Ludi Magni romains célébrés en septembre en l'honneur du Jupiter Capitolin.

Par ailleurs, nous savons avec certitude qu'il existait en Etrurie des jeux organisés dans le cadre de la confédération étrusque des douze cités, de même que la Grèce classique connaissait des jeux panhelléniques à Olympie, Delphes, Némée, ainsi qu'à l'Isthme de Corinthe. La dodécapole étrusque ne retrouvait son unité que lors de ces manifestations religieuses et spectaculaires qui devaient aussi accompagner une foire animée, un grand marché : en tout cas, elle ne réussira jamais à mettre au point une politique concertée et c'est bien ce qui a facilité la conquête romaine. A chaque fois qu'une cité étrusque appelait au secours devant les menées agressives de Rome, les autres cités toscanes avaient toujours un bon prétexte pour se défiler. A vrai dire, nous n'avons guère d'informations précises sur ces jeux étrusques, en particulier sur leur contenu, mais nous pouvons légitimement penser qu'ils avaient un programme sportif et scénique dont 
l'iconographie funéraire nous donne un reflet assez juste. Nous savons en tout cas qu'ils étaient dédiés à Tinia, le Zeus-Jupiter étrusque, le dieu principal (princeps) du panthéon toscan, célébré ici sous l'épiclèse de Voltumna, qui devait renvoyer précisément à son rôle de protecteur de la ligue des douze peuples. Ces ludi panétrusques étaient placés sous l'autorité d'un grand prêtre, d'un sacerdos et ils représentaient donc la marque la plus visible d'une unité étrusque des plus fragiles. Ils continuaient en tout cas d'être célébrés bien longtemps après la fin de l'indépendance étrusque, puisque nous les retrouvons mentionnés dans le rescrit de Spello, sous le règne de Constantin, sans doute en 337 de notre ère $^{36}$. Un fait qui montre bien à lui seul l'importance et le rayonnement de cette manifestation: il ne reste plus aux archéologues qu'à mettre au jour les vestiges du Fanum Voltumnae, de ce grand sanctuaire panétrusque que Tite-Live signale par cinq fois dans son ouvrage, sans préciser son emplacement. Mais divers indices nous ont laissé penser depuis longtemps qu'il se trouvait près de Volsinies, qui est sans conteste l'actuelle Orviéto. Or, des fouilles actuellement menées au pied de cette magnifique cité juchée sur son rocher, au lieu-dit Campo della Fiera, semblent des plus prometteuses: dans quelque temps, la Delphes étrusque sera peut-être définitivement identifiée.

\section{NOTES}

1. Voir J.-P. THUILLIER, Les Etrusques. La fin d'un mystère?, Paris, 1992 ; Id., Les Etrusques. Histoire d'un peuple, Paris, 2003. D. BRIQUEL, La civilisation étrusque, Paris, 1999.

2. J. HEURGON, La vie quotidienne chez les Etrusques, Paris, 1961, p. 241-242.

3. Sur cette question, A. HUS, "Stendhal et les Etrusques", in L'Italie préromaine et la Rome républicaine, Mélanges J. Heurgon, Rome, 1976, p. 437-469.

4. Par exemple, B. d'AGOSTINO, «Image and Society in Archaic Etruria », in JRS, 79, 1989, p. 1-10.

5. Pétrone, Satiricon, 71, 6.

6. J.-P. THUILLIER, Les jeux athlétiques dans la civilisation étrusque, Rome (BEFAR), 1985, p. 622-635.

F.-H. MASSA-PAIRAULT, La cité des Etrusques, Paris., 1996, p. 131.

7. Hérodote, $1,167$.

8. Tite-Live, $1,35,8$.

9. M. MARTELLI, La ceramica degli Etruschi. La pittura vascolare, Novare, 1987, p. 260, nº 36.

10. Sur ce dernier vase - le bucchero est la céramique noire typique de l'Etrurie - voir par exemple le catalogue de l'exposition récente du musée de Villa Giulia à Rome, Veio, Cerveteri, Vulci. Città d'Etruria a confronto, Rome, 2001, p. 170-171 (texte de M. A. RIZZO).

11. Gli Etruschi (M. TORELLI éd.), catalogue de l'exposition de Venise, Palazzo Grassi, Venise, $2000, \mathrm{n}^{\circ} 51$, p. 425 et 556.

12. On trouvera un catalogue de la plupart de ces documents dans notre thèse sur les Jeux athlétiques... (cf. note 6). Sur la peinture étrusque, voir S. STEINGRÄBER (éd.), Etruskische Wandmalerei, Stuttgard-Zürich, 1985.

13. G. SASSATELLI, "Giochi atletici in monumenti funerari di area padana ", in Spectacles sportifs et scéniques dans le monde étrusco-italique, Rome, 1993, p. 45-67.

14. J. D. BEAZLEY, Etruscan Vase-painting, Oxford, 1947, p. 2, pl. 2, 2a.

15. J.-P. THUILLIER, Les jeux athlétiques..., p. 231-254. 
16. J.-R. JANNOT, Les reliefs archaïques de Chiusi, Rome, Collection de l'Ecole Française de Rome, 1984, p. 329 sq. Id., « De l'agôn au geste rituel », in Ant. Class., 54, 1985, p. 66-75.

17. J.-P. THUILLIER, «Un relief archaïque inédit de Chiusi », in RA, 1997, p. 243-260 : sur cette image, les pugilistes sont bien accompagnés par un musicien.

18. S. STEINGRÄBER, op. cit., $n^{\circ} 92$, p. 336-7, pl. 121-126. Le nom donné à cette tombe est un peu ambigu car il semble impliquer des ressemblances avec l'agonistique grecque : en fait, il n'est lié qu'aux Jeux Olympiques modernes qui devaient se tenir à Rome deux ans après la découverte de cet hypogée couvert de fresques à sujet sportif.

19. A. PONTRANDOLFO, A. ROUVERET, Le tombe dipinte di Paestum, Modène, 1992.

20. Z. MAYANI, Les Etrusques commencent à parler, Paris, 1961, p. 315.

21. Dans les concours grecs comme Olympie, on sait que le dolichos est la course longue qui doit faire un peu moins de 5,000 $\mathrm{m}$; quant au diaulos, c'est le double stade, autrement dit une distance un peu inférieure à $400 \mathrm{~m}$.

22. J.-R. JANNOT, in JRA, 6, 1993, p. 280.

23. J.-P. THUILLIER, «La nudité athlétique (Grèce, Etrurie, Rome)», in Nikephoros, 1, 1988, p. 29-48.

24. Archölogischer Anzeiger, 1976, p. 500-501, pl. 17-20.

25. M. C. ROOT, « An Etruscan horse race from Poggio Civitate », in AJA, 77, 1973, p. 123 sq.

26. R. C. BRONSON, « Chariot racing in Etruria », in Studi in onore di L. Banti, Rome, 1965, p. 89-106. 27. J.-R. JANNOT, Reliefs archaïques... (n. 19), p. 350-355.

28. F. COARELLI, «Il Campo Marzio occidentale. Storia e topografia », in MEFRA, 89, 1977, p. 837 sq.

29. J.-P. THUILLIER, « Les desultores de l'Italie antique », in CRAI, 1989, p. 33-53.

30. J. F. BOMMELAER, Guide de Delphes. Le site, Paris-Athènes, 1991, p. 213-217.

31. G. COLONNA, «Strutture teatriformi in Etruria », in Spectacles sportifs... (n. 16), p. 321-347.

32. J.-P. THUILLIER, cf. n. 9.

33. Ovide, Amores, 3, 2 ; Ars amatoria, 1, 135-162.

34. Tite-Live, 5,1 .

35. J.-P. THUILLIER, « Mort d'un lutteur », in MEFRA, 97, 1985, p. 639-646.

36. CIL XI, 5 265. Sur cette inscription, J. GASCOU, « Le rescrit d'Hispellum », in MEFR, 79, 1967, p. 609 sq.

\section{RÉSUMÉS}

Si la civilisation étrusque fut souvent caractérisée de «mystérieuse » du fait du manque d'éléments pour la cerner, il y a un domaine que nous saisissons relativement bien : celui des jeux funéraires connus grâce aux fresques retrouvées dans les tombes étrusques. L'article procède à une analyse de la pratique sportive chez les Etrusques telle qu'elle transparaît à travers ces peintures en la situant dans le contexte de son voisinage avec les deux autres grandes civilisations antiques voisines : Rome et la Grèce.

The Etruscan civilization has often been branded "mysterious" due to a lack of physical data regarding it. Nevertheless, there is a domain which is relatively well-know: the funerary ceremonies and the games included in them can be studied thanks to the frescos found in numerous Etruscan tombs. This article proceeds to an analysis of Etruscan sport practices as they 
can be figured out thanks to these paintings. It situates them within the framework of this civilization's neighborhood with two other major civilizations of Mediterranean Antiquity: Rome and Greece.

\section{AUTEUR}

JEAN-PAUL THUILLIER

École Normale Supérieure (Paris) 